\title{
Progressive Heterotopic Heteroplasia
}

National Cancer Institute

\section{Source}

National Cancer Institute. Progressive Heterotopic Heteroplasia. NCI Thesaurus. Code C131193.

Loss of function mutation(s) in the paternal allele of the GNAS gene, encoding guanine nucleotide-binding protein $\mathrm{G}(\mathrm{s})$ subunit alpha isoforms short resulting in dermal ossification beginning in infancy, followed by increasing and extensive heterotopic bone formation in deep muscle and fascia. 